Anales del Seminario de Historia de la Filosofía

ISSNe: 1988-2564

http://dx.doi.org/10.5209/ASHF.56805

\title{
El debery lo “justo” en la ética fenomenológica: hacia una ética deontológica material
}

\author{
Pilar Fernández Beites ${ }^{1}$
}

Recibido: 13/07/2016. Aceptado: 24/03/2017

Resumen. Este ensayo defiende que la ética de los valores desarrollada a partir de la fenomenología clásica incluye una interesante teoría del deber. Desarrollando algunas tesis de Max Scheler, se ofrece aquí una noción fenomenológica de "deber" (o de "corrección", si usamos el término de Ross), que está ligado a un valor muy peculiar: el valor de lo "justo". Esto permite alejar por completo la ética fenomenológica de los valores de todo tipo de "consecuencialismo" y la sitúa en las cercanías del "deontologismo". Pero el deber de la ética fenomenológica no es un deber formal, sino fundado en el valor, de modo que podríamos hablar de un "deontologismo material", que aspira a servir de mediación entre los dos grandes paradigmas clásicos.

Palabras clave: "deontologismo material"; valor, utilitarismo; persona.

[en] Duty and "the just" in the phenomenological ethics: towards a material deontological ethics

\begin{abstract}
This article defends that ethics of values developed from classical phenomenology includes an interesting theory of duty. Developing some thesis of Max Scheler, here it is proposed a phenomenological notion of "duty" (or "the right", if we use the term of Ross), which is linked to a very peculiar value: the value of "justice". This allows to keep away the phenomenological ethics of values of all kinds of "consequentialism" and bring it closer to the "deontological" ethics. But the duty of phenomenological ethics is not formal, but founded on the value, so that we could speak of an "material deontological ethics", which aims to serve as a mediation between the two great classical paradigms.

Key words: material deontological ethics; value; utilitarianism; person.
\end{abstract}

Sumario. I. 1. Deber fundado en valor: el deber-ideal. 2. El deber-real y su diferencia respecto del deber-ideal. 3. Lo justo como correlato del deber-real (de lo correcto). 4. Lo correcto como "deber fenomenológico" fundado en lo justo. 5. Conclusión.

Cómo citar: Pilar Fernández Beites (2017): "El deber y lo "justo" en la ética fenomenológica: hacia una ética deontológica material", en Anales del Seminario de Historia de la Filosofía 34 (3), 645-661.

Para poder hablar de una "ética" de los valores, creo que no basta con disponer de una filosofía de los valores, sino que debemos acudir a una fenomenología de los valores, o mejor, a una fenomenología de la persona abierta a valores, porque sólo

\footnotetext{
$1 \quad$ Universidad Complutense de Madrid pbeites@psi.ucm.es orcid.org/0000-0002-0534-5337
} 
así se logra introducir en la teoría de los valores la necesaria perspectiva moral, que evita que dicha teoría permanezca en la antesala de la ética. Con este propósito, he desarrollado en otros ensayos una fenomenología de los valores no objetivista ${ }^{2}$, en la que los valores, lejos de reducirse a una mera tabla jerárquica objetiva al margen del sujeto, se entienden en una relación muy estrecha con la persona. Estudiando el valor desde el sujeto, ha sido posible distinguir con claridad el valor moral del resto de valores, a fin de poder situar el valor moral en el lugar que le corresponde, que no es otro que el centro de la ética. Y ahora se trata de mostrar que la fenomenología de los valores también puede hacerse cargo de la noción de "deber", que resulta decisiva en ética, y que, siguiendo a Scheler, vamos a ligar aquí a un valor muy peculiar: el valor de lo "justo".

Como es sabido, el deber es el concepto central en las éticas deontológicas, cuyo modelo lo proporciona Kant cuando en su crítica a las éticas materiales, apoyadas en la noción de "bien", propone fundar la ética en el "deber". A la ética kantiana, Scheler la denomina "ética imperativista" , entendiendo por tal aquella en la cual el deber es la única noción moral originaria, es decir, la única que logra abrir la perspectiva moral (frente a teorías prudenciales y utilitaristas, en las que una razón técnica se pone al servicio de un "bien" último, que es la felicidad). Sólo a partir de la noción de deber se introducen en la ética imperativista otras nociones derivadas, como la del "bien" (o el valor). Así nos encontramos con la "paradoja del método" reconocida por Kant, pues el "bien moral" sólo puede introducirse en la ética en un segundo momento, partiendo del imperativo categórico; y sólo en tercer lugar se cuenta con el resto de los bienes, los bienes no morales, que, bajo el término de "felicidad", se incluyen dentro del "bien supremo", cuya condición incondicionada es el bien moral.

Pues bien, frente a cualquier ética imperativista, Scheler sostiene que la noción originaria en ética es la noción de valor (que es, como el bien clásico, una noción material, pero que constituye un dato a priori capaz de escapar de las acertadas críticas kantianas). El valor es el único dato intuitivo originario y el deber es una noción derivada, que se funda en el valor. Mas no por ello se prescinde del deber en la ética de los valores. Podría parecer que es así, pues el objetivo de Scheler es superar la ética kantiana e intenta, por tanto, tomar distancia respecto a su noción central que es el deber. Pero en este ensayo veremos, prolongando las descripciones del propio Scheler, que la fenomenología de los valores ofrece una interesante teoría del deber. Se trata de lo que quizás pudiera denominarse el "deber fenomenológico", que sería un deber fundado en valores, frente al deber clásico de la ética imperativista, que es un deber formal. Para referirnos a este deber, muy distinto del kantiano, propongo utilizar la terminología sugerida por D. $\operatorname{Ross}^{4}$, en la que el deber corresponde a "lo

2 Cf. "Razón afectiva y valores. Más allá del subjetivismo y el objetivismo", Anuario filosófico 45/1 (2012) 3367. "Valores y valor moral: Scheler frente a Kant", en J. Urabayen, S. Sánchez-Migallón (ed.), Reflection on Moral in Contemporary Philosophy, Georg Olms Verlag, Hildesheim, 2014, pp. 45-76.

3 Der Formalismus in der Ethik und die materiale Wertethik, Gesammelte Werke, Band 2 (Bern und München: Francke Verlag, sechste, durchgesehene Auflage, 1980), 219. Trad. cast.: Ética (Caparrós: Madrid, 2001), 305. A partir de ahora me referiré a Der Formalismus como FE y en las citas indicaré también la página de la traducción.

4 The Right and the Good, Oxford University Press, Oxford, 1930. Trad. cast. Lo correcto y lo bueno, Sígueme, Salamanca, 1994. En el deontologismo intuicionista de Ross se supera el formalismo kantiano mediante los deberes prima facie dados de modo intuitivo, pero, como veremos, en la alternativa scheleriana dichos deberes están, a su vez, fundados en valores. 
correcto", de modo que la acción correcta es aquella que es mi deber hacer; y aquí defenderemos que el correlato de la acción correcta (debida) es el valor de lo justo ${ }^{5}$.

\section{Deber fundado en valor: el deber-ideal}

Cuando Scheler se ocupa explícitamente del deber en la sección cuarta de la segunda parte de El Formalismo ${ }^{6}$, introduce una tesis básica de cualquier ética de los valores, en la que se afirma la primacía del valor sobre el deber: "todo deber (Sollen) se funda en valores; en cambio, los valores no se fundan, de ningún modo, en el deber ideal (ideales Sollen)" (FE, 214, 298-9tr.). Y para justificarla empieza haciendo una distinción entre dos tipos de "deber": el "deber-ideal" (Sollen) y el "deber-real" (Pflicht).

El deber (Sollen) tiene un primer sentido que es el más habitual y el que también utilizamos en ética cuando hablamos del deber, por ejemplo, en la ética kantiana; se trata del sentido recogido en el término "Pflicht", que aquí traduciremos como "deber-real". Pero Scheler muestra que el deber (Sollen) también puede entenderse en un segundo sentido menos habitual, para el que propone la expresión de "deber ideal" (ideales sollen). En cuanto a la traducción, conviene precisar que, cuando Scheler emplee el término "Sollen" en este segundo sentido de deber ideal, yo lo traduciré como "deber-ideal", y cuando Scheler añada explícitamente el término "ideal" la traducción será "deber ideal". Nos damos cuenta de que Scheler no necesita siempre este añadido, porque para el deber no ideal puede utilizar Pflicht y, por tanto, el Sollen opuesto a él se reconoce, en el marco de dicha oposición, como el deber-ideal, pese a que Sollen incluye en principio las dos opciones, ya que es el verbo en el que se expresa el Pflicht (si alguien tiene el deber (Pflicht) de hacer algo, esto significa que debe (soll) hacerlo).

Pues bien, en primer lugar, Scheler va a mostrar cómo el valor funda un deberideal (y después fundará el deber-real en el deber-ideal y en una "voluntad realizadora"). Esto lo encontramos ya en la primera parte de El Formalismo, en la segunda sección, capítulo 2, donde Scheler pone de relieve las conexiones de esencias que se apoyan en el valor y las desgrana en tres axiomas. El primero de ellos se ocupa de la relación entre valor y existencia y afirma lo siguiente:

"la existencia (Existenz) de un valor positivo es en sí misma un valor positivo; la existencia de un valor negativo es un valor negativo, la inexistencia de un valor positivo es un valor negativo, la inexistencia de un valor negativo es un valor positivo" (FE, 100, 146tr. $)^{7}$.

5 Siguiendo a Scheler, voy a utilizar "justo" en su sentido amplio, como el correlato de la acción debida o correcta. Ya Aristóteles consideró este sentido amplio, que permite calificar la cobardía o la incontinencia como injustas, y lo opuso al sentido restringido, que es el de la justicia tal como hoy usamos el término, y que abarca la justicia conmutativa y distributiva (aplicada al ámbito político-jurídico). Veremos que lo justo puede confundirse con lo correcto en el lenguaje cotidiano; en concreto, el adjetivo "just" (justo) no suele sustantivizarse como "the just", sino que se utiliza la opción más cercana, que es precisamente "the right" (lo correcto); pero creo que Ross acierta al emplear esta última expresión para la corrección de la acción y aquí introduciremos el valor de "lo justo" como su correlato directo.

6 El tema del deber se plantea ya en la primera parte de El Formalismo, en la segunda sección, capítulo 2. Pero su desarrollo explícito está en la segunda parte, sección 4, en el capítulo 2 titulado "el valor y el deber ser" (en especial FE 214-226; 298-314tr.), aunque la discusión se anticipa en el capítulo 1 (FE, 192ss, 270ss.tr.)

$7 \quad$ El axioma también aparece en la primera sección de la primera parte, en el segundo capítulo, y es seguido por otros dos axiomas ligados a la voluntad, mediante los que Scheler introduce el valor moral. 
A este axioma, que Scheler atribuye a Brentano 8 , lo denominaré "axioma 1": "la existencia de un valor positivo es un valor positivo". Sabemos que Scheler considera el valor como noción originaria indefinible, que se da de modo intuitivo en un "percibir afectivo" (Fühlen), y esta noción intuitiva de valor es la que Scheler conecta con la existencia en el axioma 1. En él se afirma que la "existencia" del valor es, a su vez, valiosa (quedando claro que lo que es valioso no es la existencia sin más, sino la existencia de este valor). Y esto queda recogido en el lenguaje corriente cuando, decimos, por ejemplo, que lo valioso "merece ser", que "está bien que sea", que "es mejor que sea a que no sea". Mediante estas expresiones parecería que logramos explicar con palabras en qué consiste lo valioso y que contaríamos, pues, con una definición del valor que sería comunicable a otros por medio del lenguaje, pero lo cierto es que esa definición sólo la entiende el que ya ha intuido el valor y reconoce entonces el axioma en él fundado. El valor es un dato originario, irreductible, por tanto, al "merecer ser" y, como veremos inmediatamente, también al "deber ser". (Y recordemos que Scheler tampoco cae en el error de definir el valor diciendo que consiste en "valer", en lugar de en "ser".)

Pasemos a la segunda conexión de esencias que Scheler señala, donde ya aparece el deber bajo su forma de deber-ideal. Hemos visto que, en la primera de dichas conexiones, en la del axioma 1, se conecta el valor con la existencia del valor, y ahora se trata de conectar el valor con el deber-ideal:

"Las conexiones de esencias que existen entre valor y deber (ideal) (...). Hay en primer lugar el principio de que todo deber ha de fundarse en valores, es decir, solamente los valores deben ser y deben no ser; así como los principios que dicen que los valores positivos deben ser y los negativos no deben ser" (FE, 100, 146tr.)

A este axioma lo denominaré "axioma 2": "todo lo que tiene valor positivo debe ser". Es este axioma el que Scheler retoma en la sección cuarta, donde expresa la relación entre el deber y los valores en un texto citado parcialmente al inicio de este apartado: "Esta relación está regida fundamentalmente por dos axiomas: primero que todo lo que tiene valor positivo debe ser; segundo: todo lo que tiene valor negativo no debe ser. La conexión que así queda establecida no es una conexión recíproca, sino unilateral: todo deber (Sollen) se funda en valores; en cambio, los valores no se fundan, de ningún modo, en el deber ideal (ideales Sollen)" (FE, 214, 298-9tr.).

Pues bien, la tesis de Scheler es que el axioma 2 se apoya, a su vez, en el axioma 1: los valores deben ser (axioma 2), porque la existencia del valor es valiosa (axioma 1). En efecto, el texto anterior continúa así: "dentro de la totalidad de los valores, sólo se ligan inmediatamente con el deber-ideal aquellos valores que se fundan en el ser (o en el no-ser respectivamente) de valores, conforme a nuestros anteriores axiomas", siendo los anteriores axiomas los incluidos en el axioma 1 (según se ve

$8 \quad$ FE, 100, 148tr. En El origen del conocimiento moral (Vom Ursprung sittlicher Erkenntnis, Felix Meiner, Hamburg, 1955. Trad. cast. Tecnos, Madrid, 2002), Brentano señala varios axiomas ("reglas de preferencia evidentes"). El primero es el de oposición: preferir algo bueno a algo malo (esto se apoya en la distinción entre valor y disvalor). El segundo es preferir la existencia de algo bueno a su no existencia, y es a éste al que haría referencia Scheler, aunque bajo una formulación diferente.

$9 \quad$ Frente a las habituales malinterpretaciones que hoy se siguen produciendo, Scheler dejó muy claro que "los valores son hechos que pertenecen a un tipo determinado de experiencia"; por ello "hemos de rechazar también la afirmación de que los valores no 'son', sino que sólo 'valen' (Werte gar nicht 'sein', sondern nur 'gälten')”' (FE, 195, 274tr.). 
al proseguir la lectura). Por tanto, Scheler apoya el axioma 2 en el 1, pero lo hace de un modo que no es fácil de captar en una primera lectura. En efecto, Scheler nos está diciendo que el deber-ideal ligado al valor A no se liga inmediatamente con el valor A, sino con el valor de la "existencia" de A (valor recogido en el axioma 1), ya que sólo ese valor de la existencia de A, al que podemos denominar valor EA, es uno de esos valores que "se fundan en el ser de valores": se funda justamente en el ser (o existencia) de A. Por tanto, es el valor EA, el valor de la existencia de un valor A, lo que funda el deber-ideal de $\mathrm{A}$, y así es ya evidente que lo funda apoyándose en el axioma 1, pues sólo ese axioma 1 garantiza que la existencia del valor A es a su vez valiosa (y es por ello, por ser su existencia valiosa, por lo que A debe ser).

Como vemos, en el deber necesitamos contar con el valor de la existencia, porque el deber-ideal del valor A es un deber ser del valor A, un deber de que A "exista". El deber-ideal exige, por tanto, considerar la existencia del valor como valiosa. Y creo que Scheler tuvo esto en cuenta cuando, en el capítulo 1 de la sección cuarta, introdujo el deber-ideal y su distinción con el real, pues ya allí afirmaba que el deber-ideal exige que el valor sea considerado "respecto a un posible ser real", mientras que el deber-real es considerado "respecto a una posible voluntad realizadora". Leamos las definiciones de ambos deberes, para estudiar sus diferencias en el siguiente apartado:

"El deber está fundado siempre en un valor tal que sea considerado respecto a un posible ser real (Realsein), es decir, en esa relación. Y sólo en la medida en que esto es así proponemos hablar de un 'deber ideal (idealem Sollen)'. Frente a este existe otro 'deber' ('Sollen'), el deber-real (Pflichtsollen), que se considera, además, en relación con una posible voluntad realizadora (realisierendes Wollen) de su contenido. El primero es mentado, por ejemplo, en esta proposición: 'no debe existir lo injusto' y el segundo, en esta otra "no debes hacer lo injusto"” (FE, 194, 273tr.).

\section{El deber-real y su diferencia respecto del deber-ideal}

El deber-real, que Scheler define a partir de "una posible voluntad realizadora", es el deber en sentido clásico, el "tú debes", el deber dirigido a la voluntad de un sujeto. Se trata de una voluntad o un querer que, frente al mero deseo, se caracteriza por ser libre; y es calificada como "realizadora" porque el querer es la primera y esencial fase de la acción en la que se trata de realizar lo querido.

Lo interesante de la descripción de Scheler reside en que el deber-real ya no va a ser un mero deber formal, pues aparece al considerar los valores respecto a su realización mediante la voluntad, de modo que el tú debes significa, no que tú debes cumplir una norma (que podría ser formal), sino que tú debes realizar un valor, tú debes poner en el ser un valor, generar la existencia de un valor. Y para lograr esta descripción material del deber, Scheler va a utilizar la noción previa del deber-ideal. En concreto, Scheler defiende que el deber-real se funda en el ideal, que, a su vez, como acabamos de ver, se funda en el valor.

Para explorar la relación del deber-real con el ideal, volvemos a la sección segunda, pues en el capítulo 1, que es el que precede al de las conexiones axiomáticas, ya afirmaba Scheler lo siguiente: 
“toda 'necesidad del deber' tiene su origen en la intuición de las conexiones aprióricas entre valores, más nunca ésta en una necesidad del deber. Así, únicamente puede convertirse en 'deber-real (Pflicht)' lo que es bueno o lo que, porque es bueno (en el sentido ideal), 'debe (soll)' ser necesariamente” (FE, 93, 136tr.).

Y es esto lo que se desarrolla en el segundo capítulo de la sección cuarta, cuyo apartado b) se dedica por completo al deber-real bajo el título de "El deber normativo" (Das normative Sollen). Empezamos leyendo el mismo inicio del capítulo:

'Dentro del deber (Soll) habíamos ya distinguido el 'deber ideal' de todo otro deber que a la vez (zugleich) represente en una tendencia la exigencia y la orden. Siempre que se habla de deber o de norma no se mienta con ello el deber ideal sino su especificación en un tipo cualquiera de lo imperativo. Esta segunda clase del deber es tanto más dependiente de la primera en cuanto que todo deber-real es siempre también el deber-ser ideal de un acto de voluntad (ideale Seinsollen). Siempre que esté dado un contenido del deber ideal (idealer Sollensinhalt) y sea referido a una tendencia, él genera (ergeht von ihm) una exigencia en esa tendencia. Por consiguiente, la vivencia de tal exigencia no constituye el deber ideal, sino que es una consecuencia de él." (FE, 211, 295tr. $)^{10}$

Aquí se empieza a ver lo que añade el deber-real al ideal. En el ideal teníamos el "debe existir el valor", es bueno que exista, pero ahora añadimos la tendencia, la voluntad, y entonces en la tendencia se genera una "exigencia", la exigencia de realizar el valor, y a esa exigencia la denominamos “deber-real". El valor, que es el contenido de deber-ideal, al ligarse a una tendencia genera, pues, el deber-real, como exigencia en la tendencia.

Ya en el apartado b, Scheler repite esto mismo, pero de modo más claro: "Un deber ideal como 'lo bueno debe ser' se convierte en exigencia (Forderung), cuando su contenido es vivido a la vez en referencia a su posible realización mediante una tendencia" (FE, 218, 304tr.). Así se pasa de lo que debe ser (deber-ideal) a lo que debo hacer (deber-real), pero Scheler añade con perspicacia que este paso hay que justificarlo; es decir, que hemos de responder a la pregunta: ¿por qué debo hacer lo que debe ser? Y su respuesta consiste en señalar que "también hay un deber ideal para el ser de una determinada tendencia y querer". Esta tesis aparecía también en el texto antes citado, aunque allí pasaba quizás más desapercibida: "todo deber-real es siempre también el deber-ser ideal de un acto de voluntad". Como vemos, Scheler afirma que la exigencia propia del deber-real ha de contar con un peculiar deberideal, que es el del propio acto de voluntad, de esa "posible voluntad" que hemos hecho entrar en relación con el valor para pasar así al nivel deber-real.

Propongo entender esta tesis scheleriana del siguiente modo: como todo deberideal se apoya en un valor, el deber-ideal del acto de la voluntad ha de apoyarse en un valor, que es el valor de lo "correcto"; y, según mostraremos aquí, el correlato

10 En el cap. 1 decía: "Pero, aunque el 'deber' ideal tiene esencialmente su fundamentación en la relación de valor y realidad (Realität), el deber-real posee, sin embargo, la dirección hacia la realización (Realisierung) de un valor no real (...). El deber-real es, según esto, algo que adviene a cierto reino de valores en cuanto estos valores son considerados en la dirección de su realización mediante una posible tendencia” (FE, 195, 273tr.). 
objetivo de la voluntad correcta es el valor de lo "justo". El problema reside en que Scheler se centra en dicho correlato, en lo justo, y relega lo correcto y el deber-real a un segundo plano (para así distanciarse de la ética kantiana). Esto es precisamente lo que intentaremos evitar en las próximas páginas, pero de momento prefiero terminar este apartado insistiendo en que, mediante su teoría del deber-ideal Scheler, aboga por una tesis central en cualquier filosofía de los valores, según la cual hay una "categoría del valor", que amplía el ámbito del "ser" clásico, entendido como "ser teórico" y, sin embargo, no hay una "categoría del deber", pues el deber se funda en el valor y no el valor en el deber:

\begin{abstract}
"los valores se dan por principio como indiferentes en relación con la existencia y no existencia. En cambio, todo 'deber' está referido sin más (ohne weiteres) a la esfera de la existencia de valores (o de la no existencia) (...). El deber por consiguiente no es indiferente como el valor a la existencia, y por tanto al ser "posible" de su contenido. Todo deber es, pues, sin más (ohne weiteres) un deber ser (Seinsollen) de algo. Por lo tanto, no hay una categoría especial del 'ser-deber' (SollSeins), de tal modo que pudiera entrar como materia de esa categoría cualquier suma de valores como bueno, bello, etc. y como elemento ulterior de esa síntesis también el valor de la existencia de tales valores." (FE, 214, 299tr.)
\end{abstract}

Como vemos, dentro del ser entendido en sentido amplio, Scheler incluye la categoría del ser-valor (que se añade a la del ser-teórico), pero niega que haya una categoría del ser-deber ${ }^{11}$ (categoría que es, por cierto, la única considerada por Heidegger en su Introducción a la metafisica ${ }^{12}$, donde hace una crítica mordaz e injusta a la noción de valor, ignorando sin más las aportaciones de Scheler). El deber-ideal se funda en el valor, en cada valor hay un deber-ideal, pero estos deberes no constituyen una nueva categoría del ser, porque no son más que la relación del valor con la existencia $-\mathrm{y}$ no basta sumar a los valores el valor de la existencia para lograr la nueva categoría del deber.

\title{
3. Lo justo como correlato del deber-real (de lo correcto)
}

Hemos partido de la tesis central en la ética de los valores según la cual todo deber se funda en el valor, y ahora se trata de introducir un valor peculiar que está en relación directa con el deber: el valor de lo justo.

Como es sabido, en su tabla jerárquica de valores, Scheler sostiene que lo justo (Recht) es un valor y lo incluye en el tercer nivel de la tabla, en los valores espirituales (junto al valor estético y al valor del conocimiento). Scheler afirma que los valores de lo justo "son el último fundamento fenoménico de la idea del orden jurídico (Rechtsordung) objetivo que como tal es independiente de la idea de ley (Gesetz), de la idea del Estado... (y desde luego de toda legislación positiva)" (FE 124, 176tr.). Y quizás por esto en las exposiciones habituales se pasa por alto la importancia de

11 La traducción castellana de Soll-Seins por "ser-debido" impide entender la importante tesis que Scheler defiende.

12 Cf. los dos últimos apartados de Einführung in die Metaphysik, Max Niemeyer Verlag, Tübingen, 1998. 
este tipo de valores, pues podría parecer que aquí nos encontramos con valores sólo jurídicos. Pero, en realidad, el valor de lo justo es decisivo en el ámbito estricto de la ética (y es sólo por ello por lo que sirve para fundar el derecho positivo ${ }^{13}$ ). En concreto, aquí voy a defender que constituye el correlato objetivo de lo "correcto".

La relación de lo justo con el deber aparece ya en la primera parte de El Formalismo, donde tras los axiomas 1 y 2, Scheler formula lo que denominaré el axioma 3: "es justo todo ser de algo debido". El texto es el siguiente:

"Las conexiones de esencias que valen a priori para la relación (Verhältnis) del ser y del deber ideal y que regulan la relación (Beziehung) de éstos con el ser-justo (Rechtsein) y ser injusto. Así es justo (recht) todo ser de algo debido (deber positivo) [So ist alles Sein eines (positiv) Gesollten recht]; es injusto todo ser de algo no debido; es injusto todo no ser de algo debido; pero es justo todo no ser de algo no debido" (FE, 100, 146tr.).

Recordamos que en el axioma 1 se ponían en conexión el valor y el "ser" (la existencia de un valor es valiosa) y en el 2 se enlazaban el valor y el "deber-ideal" (los valores deben ser). Pues bien, ahora se da un paso más para introducir el serjusto; con este propósito se busca la conexión del "deber-ideal" con el "ser" y de ambos con el ser-justo, de modo que el axioma 3 afirma: "es justo todo ser de algo debido"14. Se trata, por tanto, de saber qué quiere decir Scheler cuando se refiere al ser de algo debido" (Sein eines Gesollten).

En el axioma 1 se consideraba valioso el ser del "valor", y ahora lo que se considera valioso, en el sentido peculiar de lo justo, es el ser de "algo debido". Por consiguiente, el ser de algo debido, que aparece en el axioma 3, no puede entenderse como el ser del valor, pues ese ser o existencia ya la considerábamos valiosa en el axioma 1. Lo justo ha de ser algo distinto de la existencia del valor. Aquí voy a proponer entenderlo como un peculiar "estado de cosas" (o "situación objetiva"), que consistiría en "que sea lo debido", de modo que la expresión scheleriana "el ser de lo debido" la hacemos equivalente a "que sea lo debido".

Pues bien, en una primera interpretación de este axioma 3, podríamos considerarlo como una mera reformulación del axioma 1: en el 1 se habla de la existencia del valor y en el 3 del estado de cosas que consiste en "que el valor exista". Según esta interpretación, "debido" significaría idealmente debido y el algo debido se identificaría con el valor, porque el valor es lo idealmente debido; con lo cual lo justo sería "que sea el valor debido", esto es, "que sea el valor" (pues el valor es lo debido). De este modo, el axioma 1 afirma que la existencia del valor es valiosa, el axioma 2 afirma que el valor debe ser y el axioma 3 afirma que es justo "que el valor sea"; es decir, en el axioma 1 se considera valiosa "la existencia del valor" y en el 3 se con-

13 Frente a toda teoría positivista, Scheler funda el derecho en la ética, en concreto, en el valor de lo justo, que, a su vez, funda el "orden jurídico". En nota al texto citado afirma que la ley tiene valor instrumental (konsekutivwert) para realizar el "orden jurídico": "la ley es simplemente un valor instrumental respecto al valor por sí mismo del orden jurídico: pero la ley positiva (de un Estado, por ejemplo) es el valor instrumental para el orden jurídico válido (objetivo) para ella, orden que han de realizar los legisladores y jueces" (FE, 125, 177tr.). Cf. también FE, 216, 301tr., nota.

14 Scheler habla de deber "positivo", es decir, de lo obligatorio frente a lo prohibido, porque en él se trata de introducir algo en la existencia. 
sidera valioso, en el sentido de justo, el "que sea el valor". Pero entonces el axioma 3 se convierte en una reformulación del axioma 1, en la que se cambia una expresión referente a las cosas (la existencia de A) por una referente al estado de cosas (que exista A).

Por ello creo que es más interesante una segunda interpretación. En ella entendemos el "que sea lo debido" como la realización de lo debido por parte de una voluntad, de modo que aquí el deber se convierte ya en deber-real. Lo justo es un valor peculiar sólo porque hace referencia a una voluntad realizadora, de forma que lo justo no es que exista el valor, sino que lo realice una voluntad (o lo haya realizado). Dicho con más claridad, lo justo no es que un valor llegue a ser, es decir, que se realice, sino que lo realice una voluntad. Lo justo no es que llegue a existir el valor, el idealmente debido, sino que una voluntad realice el "deber", que es el deber-ideal, pero ya convertido en real por su conexión con la voluntad realizadora. La acción o acto de realización de lo justo es lo que denominamos acto "correcto" y así lo justo es el correlato objetivo del acto correcto: lo justo es lo que debe hacerse, lo que debe realizar la voluntad.

Lo justo sería, por tanto, un valor peculiar porque hace referencia a la voluntad, de modo que si no hay referencia a la voluntad hablamos de lo valioso, pero no de lo justo. Que un valor exista es, a su vez, valioso, pero no decimos que es justo. Si el valor es o ha llegado a ser al margen de la acción humana a esto no lo denominamos justo o injusto: la muerte por enfermedad de Juan es disvaliosa, pero no injusta; lo injusto es, por ejemplo, que muera asesinado. La muerte y el sufrimiento de cientos de personas en un terremoto es altamente disvalioso, pero la injusticia o la justicia aparecen cuando consideramos la acción de las personas que quizás ayudan a los heridos y que así hacen lo justo; o bien, la acción de los que aprovechan la catástrofe para robar en las casas destrozadas y que cometen injusticia al aumentar el daño de los ya perjudicados por el terremoto. Cuando afirmamos que es injusto que haya hambre en el mundo, estamos considerando el hambre como evitable por acciones voluntarias o como causada por previas acciones voluntarias injustas, no como un mero suceso natural. Las hambrunas inevitables en tiempos sin suficiente capacidad técnica, las calificamos como disvaliosas, pero no como injustas; y cuando en esas situaciones deja de haber hambre porque ya el clima resulta favorable, consideramos que esto es valioso, pero no decimos que se haya hecho justicia. Es justa, sin embargo, la situación lograda por alguien qe decide cumplir el deber de evitar el hambre en una región, quizás mejorando los regadíos, pues en ese caso se cumple el valor de lo justo. Por utilizar un ejemplo de Scheler, es justo que A dé a B la indemnización que le debe; y podemos añadir que el que A gane en la lotería el dinero equivalente a la indemnización es valioso, pero no es ni justo ni injusto.

Así nos oponemos a la primera interpretación posible del texto de Scheler que mencionábamos más arriba, porque según ella "que exista un valor" no es sólo valioso, sino que es justo por cumplir el ser debido del valor. Pero, en este caso, y por seguir insistiendo en el asunto, a todo valor existente le tendríamos que añadir el valor de lo justo, pues la realización del deber-ideal la cumple todo valor existente y, por tanto, en todo valor existente se cumple el estado de cosas "que exista lo debido", de modo que todo valor existente sería, además, justo. Así la noción de lo justo sería redundante y creo que perdería todo su interés.

Frente a esto, estamos defendiendo que lo justo es el ser de lo debido para una voluntad. Dicho con otras palabras, interpretamos el axioma de Scheler diciendo 
que lo justo es que se realice lo debido, pero en tanto que debido. No se trata de que se realice el valor, que es lo debido (pues funda un deber-ideal), sino que se realice lo debido "en tanto que debido". Esto exige que el deber ideal haya sido captado como tal deber y para ello se necesita al sujeto, al sujeto volitivo, pero también al sujeto afectivo, que capta el valor y su deber-ideal (axioma 2), y por ello, en el nivel volitivo, quiere realizar ese deber, que es ya el deber-real, el que está en relación a una voluntad y cuyo correlato es el valor de lo justo (no el mero valor de partida, que puede pensarse al margen de la voluntad, sino el valor en relación a la voluntad).

En definitiva, en mi interpretación de lo justo pasamos del deber-ideal al real. Recordamos que cuando Scheler explica el deber-real, introduce el deber-ideal de la voluntad, que duplicaba por tanto el deber-ideal, al sumarse al de los valores iniciales. Con esto creo que nos estaba intentando decir que para pasar al deber-real no basta con reconocer el valor con su deber-ideal, sino que hay introducir una voluntad que realiza lo valioso (y que, por ello, posee un segundo deber-ideal). Esa voluntad era la que yo proponía considerar como la voluntad correcta y ahora vemos que su correlato es precisamente el valor de lo justo.

Pero he de reconocer que Scheler no se inclina por esta segunda interpretación del axioma que aquí estoy proponiendo, sino más bien por la primera, ya que limita lo justo al ámbito del deber-ideal, sin dar importancia al nivel del deber-real donde se sitúa la corrección. En efecto, cuando define lo justo a partir de las conexiones aprióricas del deber, ese deber parece coincidir con el deberideal, ya que se trata del único deber que Scheler ha introducido en ese momento de su exposición. Además, parece adoptar la primera interpretación cuando afirma “'así debe ser también’ no es más que una expresión inadecuada para una proposición de la forma siguiente: 'es justo así (es ist Recht so)'. El 'ser justo' (Rechtsein) consiste en la coincidencia del valor que - idealiter-debe ser con la existencia de este valor" (FE, 216, 301tr.). Según esto, lo justo consiste en una coincidencia del valor que debe ser idealmente con la existencia de ese valor, de modo que lo justo se aplicaría a todo valor existente, de acuerdo con la primera interpretación.

Para comprender la razón que llevó a Scheler a situar lo justo al margen del deberreal, cayendo así en la primera interpretación de su tesis, que es la menos interesante, hemos de tener en cuenta que todo su esfuerzo estriba en distanciarse de la ética de Kant, que está centrada en dicho deber-real. Y, desde luego, Scheler tiene razón en que el deber-real (la corrección) es un dato derivado, derivado respecto a lo justo, pero lo que aquí he defendido es que para llegar a lo justo no podemos prescindir de su relación con la voluntad, es decir, para llegar a lo justo hemos de situarnos en el nivel del deber-real y no sólo del ideal. Veámoslo, analizando directamente los textos de Scheler sobre la "corrección".

\section{Lo correcto como "deber fenomenológico" fundado en lo justo}

El deber-real es el deber clásico, y en este nivel Scheler habla de la "corrección", que aplica siempre a la conducta. Es frente a este deber clásico, frente al que nuestro autor propone la noción de deber-ideal, fundado en el valor, y aquí es donde introduce la noción de lo justo (Recht) frente a lo correcto (richtig). Ya en una nota al axioma 
3 que hemos estado discutiendo, Scheler liga explícitamente lo justo al deber-ideal y trata de alejarlo del deber-real, que liga a lo correcto:

"Así como el deber ideal nada tiene que ver con el deber-real (Pflicht) y la norma (Norm), tampoco tiene nada que ver lo justo con lo correcto, pues esto último se refiere solamente a una conducta (Verhalten) que es tal como la norma exige" (FE, 100, 146tr., nota).

Más adelante, en su tabla de valores, Scheler también deja clara la distinción entre los valores de lo justo y la noción de lo "correcto": "Los valores de lo justo e injusto, objetos que todavía son valores y son totalmente distintos de lo correcto (richtig) e incorrecto, es decir conforme a una ley (einem Gesetze Gemässen)" (FE, 124, 176tr.). A partir de estos textos obtenemos la definición que Scheler daría de lo correcto. Lo correcto se aplica a una conducta que es "conforme a una ley", "que es tal como la norma exige". Como vemos, Scheler liga lo correcto al deber-real, que asocia al deber clásico kantiano, y por ello lo limita al ámbito de la conducta, que es el ámbito en el que Kant sitúa el deber y la moralidad. Lo correcto es la conducta que es conforme a una norma o ley, y aquí la ley es la ley kantiana: lo correcto es el actuar "conforme al deber" descrito por Kant. Lo correcto es, pues, lo debido en el sentido clásico del deber-real.

Es comprensible que Scheler deje en segundo plano el deber-real, la corrección, porque él intenta mostrar que este deber clásico ha de fundamentarse en el valor de lo justo (ligado al deber-ideal que Scheler pone de relieve). Pero mi propuesta es dar toda su importancia al deber-real, para poder contar con una noción fenomenológica de deber (pues el deber-ideal no es lo que solemos llamar deber). A este deber fenomenológico, fundado como quiere Scheler en el valor, nos referimos con el término "corrección" utilizado en el sentido técnico de Ross. La corrección se aplica a la conducta, es decir, a la acción, pero también a la voluntad, pues es ella la que nos permite entender la conducta como "acción" en sentido estricto, como acción libre (distinta de la mera "conducta" - no libre). Y la propuesta que aquí estoy haciendo es que lo justo se aplique al correlato objetivo de la acción —o voluntad - correcta. La acción debida es la acción correcta (que funda ese segundo deber-ideal que Scheler introducía ligado la voluntad) ${ }^{15} \mathrm{y}$ en ella se realiza lo justo (que funda el deber-ideal al que se refiere en primer lugar).

Nos damos cuenta de que estas nociones de lo correcto y lo justo que aquí estamos distinguiendo, se funden, $y$, por tanto, se confunden, en las expresiones habituales en la que decimos que alguien "ha actuado correctamente" o "ha hecho lo correcto" (equivalente a la fórmula más habitual en castellano: "ha actuado bien"). Con estas palabras podemos afirmar que la acción realizada es la correcta, pero quizás lo que queramos decir es que lo correcto no es tanto la acción, sino lo realizado en ella y en este caso lo "correcto" significa lo "justo". (Y, además, es fácil dar por supuesto que la acción se hace por buenos motivos, de modo que en el hacer lo correcto se incluye el valor de lo justo y el valor moral). Esta confusión lingüística tiene su origen en el

15 Así se deja entender la afirmación de Scheler que considera que el ser de la conducta correcta es justo (siendo la corrección el valor que funda ese peculiar ser-justo): "correcta es siempre una conducta (Verhalten), y además una conducta tal cuyo ser es justo" (FE, 216, 301tr., nota). 
hecho ciertamente paradójico de que el querer no consiste en querer "ejecutar el querer" (lo que conduciría a un regreso al infinito), sino en querer "que haya lo querido". Al querer lo justo, el querer es correcto y así se cumple ese deber-ideal de la voluntad que Scheler introducía en su descripción, pero lo importante es que la corrección no es nunca el objeto del querer, sino que ella y su deber-ideal sólo es captado reflexivamente "desde fuera" del querer, pues si la exigencia de dicho deber-ideal tuviera que ejecutarse desde dentro del querer, caeríamos en un regreso al infinito: en un quererquerer (lo correcto), que sustituiría al querer-lo-justo. Dicho de modo más claro, lo querido no es el propio querer y su corrección; el querer no se ejecuta para cumplir el deber-ideal de la voluntad correcta, sino para cumplir el deber-ideal de lo justo.

Pero entremos ya en la noción de deber fenomenológico. Sabemos que en la ética scheleriana de los valores, la acción correcta puede ser definida como aquella en la que se realiza el valor más alto entre los posibles ${ }^{16}$. Y, por tanto, lo justo consistiría en "que se realice el valor más alto entre los posibles". Sin embargo, conviene profundizar ahora un poco más en esta definición, porque el "valor más alto" no puede entenderse de modo simplista, como si se tratara de acudir a la jerarquía de valores y seleccionar el más alto, para localizar en él la materia del querer. Frente a esto, creo hemos de contar muy en serio con la persona se abre al valor y que realiza el valor, pues sólo introduciendo con fuerza la persona como sujeto moral, lograremos mostrar que la ética fenomenológica de los valores queda muy lejos de cualquier utilitarismo o consecuencialismo, en las que sólo se trata de maximizar el valor de lo realizado (el valor del mundo, dejando en segundo plano al sujeto moral). A decir verdad, la ética fenomenológica se sitúa en la cercanía de las teorías deontológicas, centradas en el sujeto que experimenta el deber; pero su diferencia con estas últimas, si las limitamos a sus versiones históricas (kantiana y rossiana), reside en que la fenomenología de los valores funda ese deber en el valor, y así podríamos hablar de un "deontologismo material", que aspira a servir de mediación entre los dos grandes paradigmas clásicos. Comencemos, pues, nuestra tarea.

En primer lugar, voy a retomar la noción de "estado de cosas", al que ya me referí en la primera interpretación de lo justo ${ }^{17}$, porque al ser lo justo el correlato de una

16 Aunque Scheler no formula explícitamente esta definición, porque él afirma estar definiendo así el acto "moralmente bueno" (es decir, Scheler da por supuesto que si se elige el valor más alto se hace por ser el más alto, pero en otro lugar he mostrado la necesidad de distinguir ambas nociones).

17 Reinach introduce con fuerza la noción de estado de cosas cuando afirma que lo valioso se aplica a cosas y lo justo sólo a estados de cosas; y, además, defiende una tesis semejante a la de Scheler (con la diferencia de que Scheler entiende lo justo como un tipo de valor): "Todo deber moral tiene como condición necesaria, si bien no suficiente, el ser-justo (Rechtheit) moral de los estados de cosas; en particular (insbesondere), presupone que la existencia de la conducta (Verhalte) de una determinada persona - conducta que constituye el contenido de su deber-, sea en sí o como consecuencia de la corrección de otros estados de cosas conectados con ella, moralmente justa (recht)". Y añade en nota: "Distinguimos rigurosamente el ser-justo moral, que puede convenir a los estados de cosas y sólo a ellos, del valor moral de personas, acciones, acto, etc. De este modo, se delimitan dos esferas de la Ética que están ligadas entre sí por relaciones esenciales inmediatamente evidentes. Así es moralmente justo que exista un objeto moralmente valioso (...) Además, la realización de un estado de cosas éticamente justo es moralmente valiosa." (Die apriorische Grundlagen des bürgerlichen Rechtes, en Sämtliche Werke, I, Philosophia Verlag, München, 1989, pp. 152-3. Trad. cast. Los Fundamentos a priori del Derecho Civil, Ed. Comares, Granada, 2010, 34-5). Como vemos, Reinach afirma que el deber se apoya en el ser-justo de los estados de cosas (Reinach califica lo justo como moral, al igual que el valor de los objetos, quizás para indicar que estamos en una investigación moral y no legal). Dice que "en particular" el deber presupone que sea justa la existencia de la conducta (en el inicio del segundo capítulo, Reinach ya sólo se refiere a esto) y así Reinach parece añadir la "corrección" de la conducta al "ser-justo" del estado de cosas (el "contenido" del deber es la conducta, que es correcta, y lo justo sería el "objeto" del deber), pero el problema reside en que no distingue 
voluntad, a él le va corresponder necesariamente ese peculiar nivel ontológico que designamos como "estado de cosas", que es lo que el sujeto quiere realizar. En efecto, si pensamos, por ejemplo, en el fin de la acción, ese fin no es nunca una cosa: el fin del que roba no es el dinero, sino lograr un nuevo estado de cosas en el que el dinero esté en su poder y no en el del banco atracado. E incluso cuando la acción es la "fabricación" de una cosa, el fin de la acción no es la cosa, sino "que exista la cosa", por ejemplo, el jarrón; el presidente del Banco Central Europeo puede proponerse como fin "que exista más dinero", pero, al igual que sucede con el ladrón, tampoco él puede proponerse como fin "el dinero".

En definitiva, mientras los valores en general se atribuyen a las cosas, el valor peculiar de lo justo se atribuye necesariamente a un estado de cosas. Por tanto, hemos de introducir en el nivel del valor la noción equivalente a la de estado de cosas que reconocemos en el nivel teórico. Se trata de lo que Scheler denomina "Wertverhalt", que hace juego con "Sachverhalt" (estado de cosas o situación objetiva) y que propongo traducir por "estado de valores" o "situación objetiva de valores"18. Y así lo justo podemos definirlo ya de modo más preciso como un peculiar "estado de valores", que incluye la relación con la voluntad del sujeto. Lo justo es el estado de valores en el que se articulan los valores de partida al ponerse en relación con la voluntad.

Si retomamos las descripciones de Scheler, veíamos que el deber-real se apoya en los valores de partida y en su deber-ideal: ellos, en su relación con la existencia, generan el deber-ideal y en su contacto con la voluntad generan el deber-real. Los valores de partida son ese "contenido" del deber-ideal, a partir del cual se generará el deber-real ("el deber ideal se convierte en exigencia, cuando su contenido es vivido a la vez en referencia a su posible realización"). Y lo que aquí estoy sugiriendo es que lo justo son esos mismos valores, pero "reestructurados" en su relación con la voluntad. Así pasan de ser valores (contenido del deber-ideal) a constituir el valor de lo justo (contenido del deber-real). Cuando los valores se ponen en contacto con la voluntad ellos han de ser reestructurados en el valor lo justo: lo justo es ya el objeto del deber-real, mientras que los valores fundaban sólo un deber-ideal (cada uno el suyo). Lo justo es así un valor complejo que incluye, a su vez, distintos valores en relación a la voluntad ${ }^{19}$.

Intentemos describir más de cerca este peculiar estado de valores. Para llegar a lo justo ponemos los valores en relación con la persona: con la voluntad realizadora,

\footnotetext{
ambos términos, pues para la corrección de la conducta también utiliza "justo". En la nota, Reinach diferencia lo justo del valor moral y para precisar su relación afirma, en primer lugar, que es justo que exista un objeto valioso, lo cual correspondería al axioma 3 de Scheler en la primera interpretación. Además, considera que la realización de un estado de cosas justo es moralmente valiosa, lo que correspondería a nuestra interpretación del axioma 3 si suponemos que el valor es la corrección de la voluntad realizadora y que Reinach, como Scheler, liga dicha corrección al valor moral en sentido estricto. Quizás conviene añadir que, en Die Grundbegriffe der Ethik (Sämtliche Werke I. op. cit., Teil II. Nachgelassene Texte, p. 336), Reinach parece reconocer, tal como haremos nosotros más adelante, que, frente al valor intrínseco de lo justo (recht), lo correcto (richtig) sólo posee un valor instrumental; pero Reinach sigue utilizando los términos indistintamente, es decir, sin fijar correcto para el querer y justo para lo querido: "Un querer no es en sí correcto (richtig) (...) Es justo (recht) en tanto que es justo lo que es querido, pero no en sí (...). Traspasamos de lo querido al querer". Sobre la teoría del Derecho de Reinach, cf. Marta Albert Márquez, Derecho y valor, Encuentro, Madrid, 2004.

18 FE, 141, 201tr. La traducción castellana ("contenido de valor", que hace juego con "contenido objetivo") impide entender la tesis de Scheler.

19 Así podría entenderse la afirmación difícil de que "el ser injusto y ser justo mismos son depositarios de valores, por tanto, no son, eo ipso, origen de ellos" (FE, 216, 301tr., nota).
} 
es decir, con el sujeto libre que puede actuar. En primer lugar, como este sujeto es finito, lo justo ya no puede abarcar todos los valores, que son infinitos, sino sólo los realizables por el sujeto; en términos clásicos, lo justo ha de ser "posible para mí". Por ello al definir lo justo limitamos el ámbito de los valores a los valores "posibles". Pero esta primera condición no es suficiente, realizar lo justo no puede consistir simplemente en realizar "un valor posible", pues se trata, más bien, de "elegir" entre múltiples valores posibles, ya que ante el sujeto que va a actuar se abren distintas posibilidades $\mathrm{y}$, por tanto, tiene que elegir entre ellas (el campo de posibilidades puede ser más o menos amplio, pero incluso si las posibilidades se limitan a una, al menos hay la posibilidad de elegir esa una o no elegirla; es decir, en la voluntad no podemos prescindir de la elección). Entonces ya no basta con realizar un valor posible "cualquiera", sino que hay que añadir la exigencia de que se realice el valor "más alto" entre dichos valores posibles, que es lo recogido en la definición de lo justo ${ }^{20}$.

Pero, como he indicado, aquí queremos alejarnos de una versión simplificada en la que al valor más alto de los dados lo denominaríamos el valor de lo justo. Se trata de precisar que lo justo no es un valor, sino un "estado de valores": aquél que tiene un "valor global" más alto que los otros múltiples estados de valores alternativos que se ofrecen ante la voluntad del sujeto. De este modo, llegamos al punto más difícil de la descripción, que consiste en que el valor global del estado de valores no puede entenderse, a su vez, como una mera suma de los valores, ni siquiera como un todo gestáltico de valores, ya que aquí lo decisivo es la relación de esos valores con el sujeto moral. Por tanto, para definir lo justo es necesario "ponderar" los valores en su relación con el sujeto moral, y lo justo coincidirá con la más alta de las diversas sumas ponderadas $^{21}$. Veámoslo, aunque sea muy sucintamente.

En primer lugar, desde una teoría fenomenológica de la acción, es el sujeto moral el que fija la estructura de la acción mediante la "intencionalidad" que proporciona

20 Este paso nada sencillo que va de lo valioso a lo correcto ya lo intenta dar Brentano, en El origen del conocimiento moral, que es donde se introduce por vez primera la noción de valor ligada al ámbito afectivo (cf. Juan Miguel Palacios, "Brentano en las inmediaciones del valor", Bondad moral e inteligencia ética. Nueve ensayos de la ética de los valores, Encuentro, Madrid, 2008, 31-44). Brentano se da cuenta de que en la acción ética no basta con recurrir al "bien" (el valor), sino que hemos de contar con lo "mejor", pues en ella se trata "realizar lo mejor entre lo accesible" (lo accesible al sujeto). Éste es el imperativo brentaniano que intenta sustituir al imperativo kantiano; pero, en realidad, este imperativo se ocupa de lo "correcto" y es por ello por lo que hemos de pasar del bien a lo mejor, que en Brentano (todavía utilitarista), es la "suma armónica" de los distintos bienes, que ya reconoce nuestro autor frente al clásico utilitarismo hedonista. Por su parte, Scheler cuenta con la jerarquía de valores, que impide considerar lo mejor como una mera suma armónica, pero para acabar de alejarse del utilitarismo veremos que hay que articular dichos valores jerárquicos en su relación con el sujeto moral.

${ }^{21}$ El deber-real corresponde al valor global más alto y así, en una situación concreta, logra ser el "vencedor" entre los múltiples deberes-ideales, pero, aunque sólo gana el valor más alto, los más bajos siguen poseyendo su deber-ideal, que sería deber-real de no tener que competir con otro más fuerte en la actual situación compleja, es decir, que sería deber-real en una situación más simple. Esto nos permite ligar la fenomenología de los valores con la propuesta de Ross, pues los deberes "prima facie" de Ross pueden entenderse como los deberes reales "típicos", que son los dados en situaciones simples (fáciles, por tanto, de tipificar). Ellos son los que entran en conflicto cuando la situación concreta es compleja e implica a varios de esos deberes; en ese caso sólo el deber más fuerte (el fundado en el valor global más alto) es el que se convierte en lo que Ross denomina el "deber real", que coincide con el scheleriano. Como vemos, la tesis de Scheler es que los deberes prima facie de Ross se fundan finalmente en valores. Por dar alguna indicación, en el caso central y difícil de la promesa, las acciones del agente moral, en este caso lingüísticas, generan relaciones interpersonales a las que corresponden peculiares "estados de cosas" (esta dirección es la explorada por Reinach, que fue pionero en el descubrimiento del carácter performativo del lenguaje) y, por tanto, podríamos señalar ciertos "estados de valores" también peculiares (valor del conocimiento de "verdades futuras" dependientes de la acción del que promete...), desde los que se podría fundamentar el deber de cumplir la promesa. 
su sentido a los movimientos corporales. En la acción configurada intencionalmente, los valores lo son, o bien, de los "fines" y "medios" (consecuencias "queridas" de los movimientos corporales), o bien, de los "efectos secundarios" (consecuencias de los movimientos corporales tan directas como el fin y los medios, pero que son "no queridas", aunque sí "previstas"), y debido a esto poseen un peso distinto en el valor final de lo justo. En efecto, el fin - y los medios como fines subordinados- definen esencialmente la acción pues determinan el qué de la acción, y por ello su peso es muy superior al de los efectos secundarios, que, al ser no queridos, configuran la acción de modo accidental (esto permite diferenciar, por ejemplo, el homicidio intencional del homicidio imprudente). Además, hay el resto de consecuencias de los movimientos corporales que ya no configuran la acción en ningún sentido, y que son en número infinito; ellas son meras "consecuencias de la acción" y aunque, desde luego, sean en sí mismas valiosas o disvaliosas, no intervienen en el valor global de lo justo (en el entendido de que el "deber de prever" varía según el tipo de acción, pues la negatividad de los efectos secundarios puede manifestarse a muy largo plazo, como en los medicamentos, las bombas atómicas o los alimentos transgénicos).

En segundo lugar, como en la definición de lo justo se incluye el sujeto que actúa, va a resultar decisiva su relación personal respecto a otros sujetos implicados. Esto permite hablar, como quiere Ross frente al utilitarismo, del "carácter altamente personal del deber" (por ejemplo, el deber de una madre respecto a su hijo es distinto que su deber respecto a los hijos de su vecino).

En tercer lugar, de acuerdo con la tesis central scheleriana sobre el ordo amoris, el sujeto finito se abre al ámbito del valor de una forma estrictamente individual, de modo que los valores de partida entre los que elige la persona son ya distintos para cada individuo (el artista cuenta con valores que ni siquiera aparecen ante el científico) y así cada sujeto experimenta una exigencia por parte del ámbito valorativo que es distinta de la que experimentaría otro sujeto situado en el mismo lugar espaciotemporal; pero que es igualmente estricta y vinculante, pues la objetividad del deber no depende de su universalidad. El ordo amoris individual es el que se objetiva parcialmente en los roles sociales, por ejemplo, en el estatus profesional (mediante el que distinguimos al artista del científico), pero no se agota en dicha objetivación, pues admite los matices que quedan desdibujados en el rol social y que hacen que lo debido éticamente pueda no coincidir con el deber profesional, en el que se fijan ciertos mínimos exigibles de modo universal (dicho de otro modo, la realización profesional se inserta en el proyecto más amplio de realización vital, que es el fijado por el ordo amoris).

Teniendo esto en cuenta, podemos alejar a la ética fenomenológica de todo consecuencialismo, que suma los valores de las infinitas consecuencias de la acción y deja fuera de consideración al sujeto que actúa, perdiendo así la perspectiva moral. En la fenomenología de los valores la corrección se sitúa en la misma acción (no en el valor de las consecuencias), pero frente a la tesis formalista que funda la corrección en la universalidad del imperativo categórico, la ética material la funda en los valores que son el correlato de la acción, en concreto, en el valor complejo de lo justo.

Ya podemos ir recapitulando. Nuestro ensayo ha partido de la tesis central en la ética de los valores según la cual todo deber se funda en el valor y hemos descubierto el valor peculiar de lo justo como el ser de algo "debido". Podría parecer que así Scheler reconocería que un valor central en ética, el de lo justo, ha de apoyarse en "lo debido", en el deber, y estaría, pues, renunciando a su tesis de la prioridad del valor 
sobre el deber. Pero, en realidad, no es así. Lo justo no se funda en el deber-real, sino que, a la inversa, es el fundamento de dicho deber, y lo justo tampoco se funda en el deber-ideal, pues lo justo es una reestructuración de los valores iniciales del mundo (que son los que fundan deberes-ideales), en la que sólo se necesita que esos valores se pongan en relación con la voluntad. La voluntad o la libertad del sujeto, que es lo que permite el paso de los valores del mundo al deber del sujeto, representa un dato ineludible en ética, pero lo decisivo es que ella misma no es un dato moral. El dato moral sí podría ser, desde luego, la "ley" kantiana que da su "positividad" a la libertad (libertad positiva frente a mera libertad negativa), pero Scheler muestra que esa ley ha de fundarse en los valores (la universalidad como tal no puede aportar la positividad, porque tampoco ella es un dato moral).

\section{Conclusión}

La ética fenomenológica de los valores aquí desarrollada sostiene que la perspectiva moral no la proporciona el deber, sino el valor, pero el valor en relación a la persona. En un primer momento podría dar la impresión de que la noción de valor se queda en la antesala de la ética, pues la moralidad sólo surgiría con los "deberes" que ha cumplir el sujeto, ya que sólo la acción debida o correcta del sujeto, y no los valores del mundo externo, plantean la cuestión de la moralidad. A esto puede objetarse de inmediato que el deber, que parece abrir la perspectiva moral, no es en realidad el dato moral central, porque, según reconoció el propio Kant, el centro de la moralidad lo ocupa un valor, el "valor moral", que se obtiene, no al realizar lo correcto, sino al realizarlo por buenos motivos ("buena voluntad"). Pero, aun haciendo esta necesaria precisión, podríamos seguir afirmando que la perspectiva moral sólo se ha abierto con la noción de deber, ya que el buen motivo consistiría, como dice Kant, en hacer el deber "por deber" (aus Pflicht).

Pues bien, frente a esto, hemos defendido con Scheler que el deber se funda, a su vez, en el valor: el deber-real en el deber-ideal y éste en el valor de lo justo (que reestructura los valores de partida del mundo) y, por tanto, el valor moral que centra la ética no consiste en hacer el deber por deber, sino en realizar lo justo por ser justo (y así el valor moral se amplía "más acá" de la acción “externa”, pues abarca la "actitud ante el valor", pero en esto ya no puedo entrar aquí). En efecto, el valor moral se liga al deber porque para ser moralmente bueno he de cumplir mi deber (o mejor dicho, lo que creo que es mi deber) y, además, he de hacerlo por buenos motivos, pero como el deber se funda en los valores, son esos valores los que pueden configurar los buenos motivos a partir de los cuales se genera el valor moral (cuando el sujeto los realiza libremente por su carácter valioso), de modo que el valor moral, que constituye el núcleo de la perspectiva ética, no depende del deber, sino del valor.

Por tanto, la experiencia moral comienza y acaba en el valor: se inicia en los valores de partida a los que se enfrenta el sujeto libre cuando emprende su aventura moral y culmina en el valor moral. El valor moral es, sin duda alguna, el centro de la moralidad, pero él sólo puede darse al final y "a la espalda" del acto. Al inicio y "de frente" se dan, más bien, los valores de partida, que son los encargados de iniciar la aventura moral, reestructurándose en el valor de lo justo. Los denominamos valores no morales para distinguirlos del valor moral, pero sostenemos con Scheler (frente a Kant) que son valores en el mismo sentido que el valor moral (que es el valor supre- 
mo) y así resulta comprensible que ellos se conviertan en los buenos motivos capaces de iniciar la vida moral que culmina en el valor moral22.

Teniendo esto en cuenta, el deber ocuparía un lugar intermedio en la vida moral, al situarse entre los valores no morales y el valor moral (según el esquema: valor no moral — deber - valor moral). El ser "debido" o "correcto" de la acción (o el querer) lo hemos entendido, a su vez, como un valor (que por ello funda ese deber-ideal de la voluntad al que se refería Scheler), pero ya podemos precisar que ese valor no es un valor intrínseco, sino instrumental, pues la corrección de una acción sólo tiene valor en tanto que realiza lo justo (y así puede generar el valor moral a la espalda del acto). Es decir, en la acción tiene valor intrínseco, o bien, lo realizado (valor de lo justo, que reestructura valores no morales), o bien, el sujeto o la acción en tanto que se obra por buenos motivos (valor moral), pero la corrección misma de la acción tiene un valor sólo instrumental. Como afirma el propio Ross: "cualquier valor intrínseco (...) que pueda tener la acción, lo debe a la naturaleza de su motivo y no a que el acto sea correcto o incorrecto; y cualquier valor que ésta tenga independientemente de su motivo es valor instrumental, es decir, no es en absoluto bondad, sino la propiedad de producir algo que es bueno"23.

En definitiva, lo debido posee un valor instrumental, que remite a los valores intrínsecos de lo justo (valor del mundo en relación con el sujeto) y de lo moralmente bueno (valor supremo, que lo es del sujeto mismo y nunca del mundo), y es en estos valores donde reside la perspectiva moral - ausente en la universalidad de un deber formal-. Aunque no suela señalarse en las exposiciones habituales, dichos valores se incluyen en la tabla jerárquica de valores propuesta por Scheler, que logra incorporar así la perspectiva moral, porque, según mostré en otro lugar, el valor moral corresponde a los valores de lo santo, que no deben identificarse con los "valores religiosos" y, como aquí he intentado poner de relieve, el valor de lo justo tampoco debería identificarse con los "valores jurídicos", salvo que estemos dispuestos a perder toda la potencialidad de la brillante propuesta que hizo Scheler hace más de un siglo.

22 La diferencia socrática entre cometer injusticia y padecer injusticia, la hemos expresado nosotros como la diferencia entre realizar una acción incorrecta, que trae al ser lo injusto, y el que exista lo injusto o, mejor dicho, lo disvalioso, es decir, el elemento disvalioso de la situación que padece la persona con la que se comete la injusticia. Y, como ya vio Sócrates, aquí el disvalor máximo, el peor de los males, es el disvalor moral, ligado al cometer injusticia (y, por tanto, al disvalor de lo injusto) y no al padecerla (existencia de algo disvalioso).

23 The Right and the Good, 133, 150tr. 